\title{
Temperature Insensitive Waveguide Interferometer Based on Subwavelength Gratings
}

\author{
Umair A. Korai \\ Dept. of Electronic and Electrical \\ Engineering, \\ University of Strathclyde, \\ Glasgow, United Kingdom \\ umair.korai@strath.ac.uk \\ Ivan Glesk \\ Dept. of Electronic and Electrical \\ Engineering, \\ University of Strathclyde, \\ Glasgow, United Kingdom \\ Ivan.glesk@strath.ac.uk
}

\author{
Alaine H. Bermello \\ Institute of Optics, \\ Spanish National Research Council, \\ Madrid, Spain \\ alaine.herrero@csic.es
}

\author{
Aitor V. Velasco \\ Institute of Optics, \\ Spanish National Research Council, \\ Madrid, Spain \\ a.villafranca@csic.es
}

\author{
Michael J. Strain \\ Institute of Photonics, Dept. of Physics, \\ University of Strathclyde, \\ Glasgow, United Kingdom \\ Michael.strain@strath.ac.uk
}

\begin{abstract}
We present a design for a temperature insensitive Mach-Zehnder interferometer in which temperaturecompensating segment is achieved through tailored subwavelength gratings. By engineering the thermal response and the relative length of this segment, an overall temperature insensitivity below $\pm 4 \mathrm{pm} /{ }^{\circ} \mathrm{K}$ is predicted for $100 \mathrm{~nm}$ bandwidth around $1550 \mathrm{~nm}$.
\end{abstract}

Keywords - Integrated optics, athermal design, spectroscopy, thermo-optic effects, silicon-on-insulator

\section{INTRODUCTION}

Silicon is a very attractive material for fabrication of integrated photonic devices, as it offers reduced fabrication cost and very high refractive index which enables high mode confinement and. One of the most challenging obstacles of using this material is its high thermal dependency, driven by the large thermo-optic coefficient (TOC) of silicon, i.e. $1.86 \times 10^{-4}[1]$, which is almost ten times greater than silica. In particular, when fabricating resonators and interferometers with long propagation lengths and critical coupling conditions [2], small temperature variation will significantly affect the effective index of refraction of silicon waveguides, and therefore, the resonant wavelength, coupling response and overall performance of these devices.

In integrated optics, there are several ways of dealing with a temperature induced wavelength shift. For example, thermoelectric coolers can be integrated on the chip, but they require additional power and controlling elements. This approach is not suitable when dealing with a very large scale integration [3]. Negative TOC materials, such as polymers or titanium oxide $\left(\mathrm{TiO}_{2}\right)$, have also been used as cladding to compensate for the positive TOC of silicon [4]-[5]. The use of wider and narrower waveguides in MZI has also been reported to help to overcome the temperature dependency [6]-[7]. However, all the aforementioned solutions present either bandwidth limitations, add to fabrication complexity or are over-sensitive to fabrication errors

In this work, we present a novel temperature insensitive silicon-on-insulator (SOI) MZI which combines wire waveguide and subwavelength grating (SWG) waveguide segments. By engineering their thermal response and relative length, an overall temperature insensitivity below $\pm 4 \mathrm{pm} /{ }^{\circ} \mathrm{K}$ is attained in a $100 \mathrm{~nm}$ bandwidth around $1550 \mathrm{~nm}$.

\section{OPERATION PRINCIPLE AND DEVICE MODELLING}

SWG waveguides are periodic dispositions of alternating core and cladding materials with a pitch much smaller than is the wavelength of the guided light [8]. In SWG waveguides, diffractive effects are cancelled, generating a homogeneous metamaterial which combines the optical and thermooptic properties of its constituents. The schematic diagram of the SWG waveguide is shown in Fig. 1. Effective index and thermal response of the resulted waveguide can hence by adjusted by varying the duty cycle (DC), (defined as $a / \Lambda$, of the waveguide, where $a$ is the length of the silicon segment and $\Lambda$ is the period of the grating, known also as pitch), as well as its period and width.

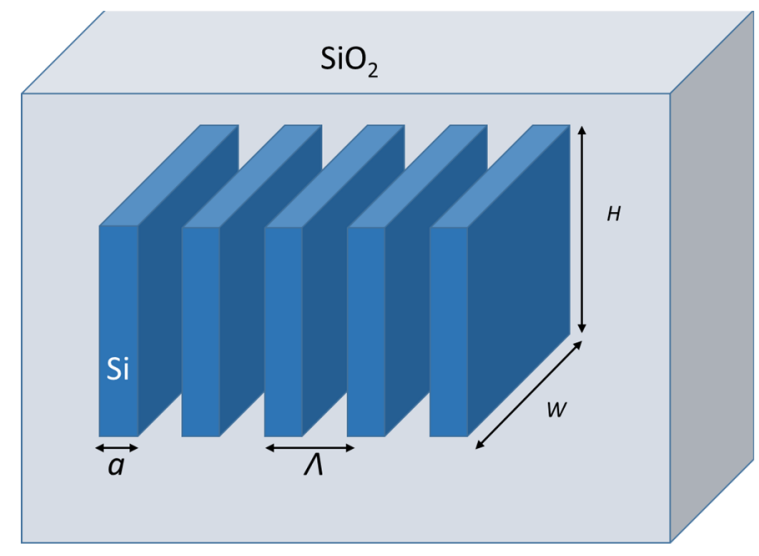

Fig. 1. Schematic diagram of silicon based subwavelength grating waveguide. $a$ - length of silicon segment, $A$ - pitch of the gratings, $w$ - width and $h$-height of the gratings

The schematic of the proposed temperature insensitive MZI is shown in Fig. 2. The basic idea behind achieving the device temperature insensitivity is to cancel the temperature effect introduce in the first arm $\left(A_{l}\right)$ with the temperature effect introduced in the second arm $\left(A_{2}\right)$. For this purpose, 
careful consideration of the widths, DC, and lengths of the waveguides in both arms is necessary.

In our proposed device, we used a wire waveguide in $A_{I}$ whereas in $A_{2}$, a combination of the wire waveguide and the SWG waveguide. We have also introduced four tapers $\left(T_{1}-T_{4}\right)$ to adiabatically adapt the mode from the narrower waveguide (width $W_{1}$ ) to the wider waveguide (width $W_{2}$ ) and vice versa In addition, SWG tapers $\left(T_{5}, T_{6}\right)$ were included to adapt the mode from the wire waveguide to the SWG waveguide (width $\left.W_{3}\right)$. In order to cancel the thermal dependence of these SWG tapers $\left(T_{5}, T_{6}\right)$ on arm $A_{2}$, two identical SWG tapers $\left(T_{7}, T_{8}\right)$ were included in a back-to-back configuration in $A_{l}$. A standard waveguide height of $220 \mathrm{~nm}$ has been considered in all elements.

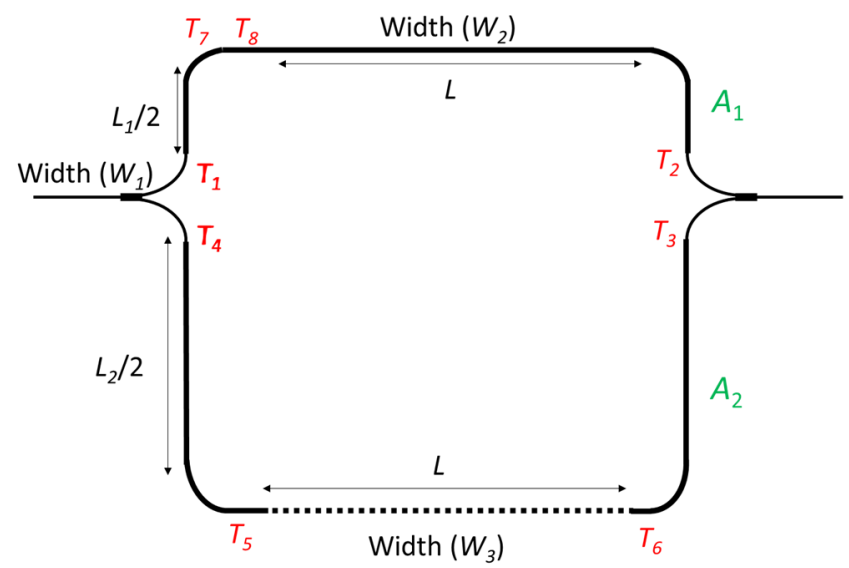

Fig. 2. Novel temperature insensitive MZI device based on the combination of SWG and wire waveguide.

The transmitting wavelength of the proposed temperature insensitive MZI is given as:

$$
m \lambda_{0}=n_{e f f}\left(W_{2}\right) \cdot \Delta L+\Delta n_{e f f} \cdot L
$$

where $n_{\text {eff }}\left(W_{2}\right)$ is the effective index of the waveguide $W_{2}, \Delta L=L_{2}-L_{1}$, is the path length difference between both arms and $L$ is the common length of both waveguides (wire and SWG), $m$ is the interference order, which can be an integer to provide constructive interference or a half-integer to provide destructive interference for a given wavelength of operation $\left(\lambda_{0}\right) . \Delta n_{e f f}=n_{e f f}\left(W_{3}\right)-n_{e f f}\left(W_{2}\right)$, is the effective index difference between the effective indices of SWG waveguide at width $W_{3}$ and wire waveguide of width $W_{2}$. When considering waveguides, the interference order of the MZI is modified as:

$$
M=m-\Delta L \frac{d n_{e f f}}{d \lambda}-L \frac{d\left(\Delta n_{e f f}\right)}{d \lambda}
$$

Thus, the overall temperature sensitivity of the athermal MZI at any specific wavelength $\left(\lambda_{0}\right)$ is given by [7]. It can be deduced from (3) that by carefully considering the effective index and the waveguides length, the temperature sensitivity can be reduced to zero.

$$
\frac{\Delta \lambda 0}{\Delta T}=\frac{\Delta L \frac{d n_{e f f}}{d T}+L \frac{d \Delta n_{e f f}}{d T}}{M}
$$

Based on this model, we optimized the temperature sensitivity and spectral response of the SWG structure through Bloch modes computation using band-structure calculations in Lumerical FDTD solution. In particular, we have considered parameters of SWG as follows, the width $\left(W_{3}\right)$ ranging from 400 to $600 \mathrm{~nm}$, the duty cycle of $50 \%$ for ease of fabrication (but to get the optimal results, the range of $40 \%$ to $60 \%$ has been also considered in our simulations). The pitch is very important parameters in the SWG waveguide design and has to be chosen very carefully. In order to avoid Bragg effects, the pitch, $\Lambda=220 \mathrm{~nm}$, has been used in our simulations. For the wire waveguide, the width $\left(W_{2}\right)$ ranges from 350 to 600 $\mathrm{nm}$. The Y-splitter waveguide width $\left(W_{l}\right)$ has an SOI standard single mode width of $500 \mathrm{~nm}$. We have considered the TOC coefficient for $\mathrm{Si}$ as $1.86 \times 10^{-4}$ and TOC for silica as $1 \times 10^{-5}$.

\section{RESULTS AND DISCUSSION}

By solving (1) - (3), we found that variations in the waveguides common length less than $L= \pm 0.355 \mathrm{~mm}$ keeps the temperature sensitivity within $\pm 1 \mathrm{pm} /{ }^{\circ} \mathrm{K}$. The performance of this novel device was then analysed in terms of the optical path imbalance and the overall temperature sensitivity in the wavelength region of $1500-1600 \mathrm{~nm}$. By further analysing (1) - (3), we found that for $W_{2}=600 \mathrm{~nm}, W_{3}$ $=600 \mathrm{~nm}$ and $D C=60 \%, L=50.62 \mathrm{~mm}$ and $\Delta L=23.55 \mathrm{~mm}$, the temperature dependent wavelength shift will become zero. Fig. 3 shows the temperature sensitivity response using the above parameters.

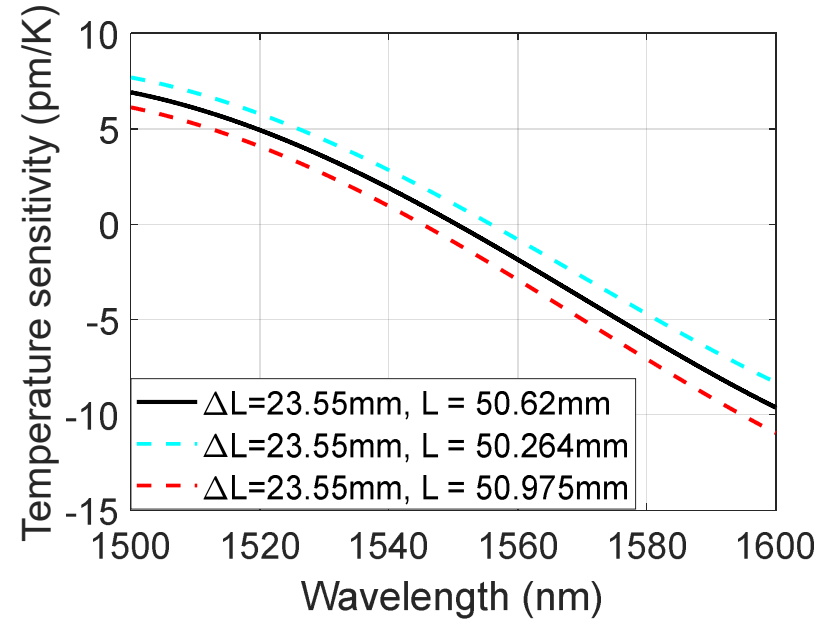

Fig. 3. The spectral response of novel temperature insensitive MZI device when using parameters: $\mathrm{W} 2=600 \mathrm{~nm}, \mathrm{~W} 3=600 \mathrm{~nm}, \mathrm{DC}=60 \%$. 
It can be seen that any variations of the waveguide length (L) smaller than $\pm 0.355 \mathrm{~mm}$ will maintain the central wavelength temperature dependent wavelength shift within \pm $1 \mathrm{pm} /{ }^{\circ} \mathrm{K}$.

Our next investigations will show that the residual temperature sensitivity of the novel $\mathrm{MZ}$ structure in the 1500 $-1600 \mathrm{~nm}$ wavelength band can be further reduced by refining its geometrical parameters. We found, that by reducing the width and DC of the SWG waveguide, the overall temperature sensitivity can be further reduced. This approach will require larger $\Delta L$ and $L$ to maintain the threshold of variations of $L$ within $\pm 0.355 \mathrm{~mm}$. Therefore, a trade-off is needed between the optical path imbalance and the overall temperature sensitivity. In order to investigate the interplay between temperature sensitivity and optical path imbalance, the following three cases were considered:

1. $W_{2}=400 \mathrm{~nm}, W_{3}=400 \mathrm{~nm}, D C=40 \%$, $L=75.1 \mathrm{~mm}, \Delta L=64.4 \mathrm{~mm}$.

2. $W_{2}=500 \mathrm{~nm}, W_{3}=500 \mathrm{~nm}, D C=50 \%$, $L=62 \mathrm{~mm}, \Delta L=42.5 \mathrm{~mm}$.

3. $W_{2}=600 \mathrm{~nm}, W_{3}=600 \mathrm{~nm}, D C=60 \%$, $L=50.6 \mathrm{~mm}, \Delta L=23.5 \mathrm{~mm}$

The temperature sensitivity response of all three above cases is shown in Fig. 4. It can be seen that with a lower width and duty cycle values, the overall temperature sensitivity in the entire $1500-1600 \mathrm{~nm}$ region is lower but requires large lengths of $\Delta L \& L$ to keep the variation of $L$ within \pm 0.355 $\mathrm{mm}$, meaning the optical path imbalance is high. Whereas, if the width and $D C$ of the SWG are increased, the overall temperature sensitivity is also increased but requires shorter lengths resulting a lower optical path imbalance.

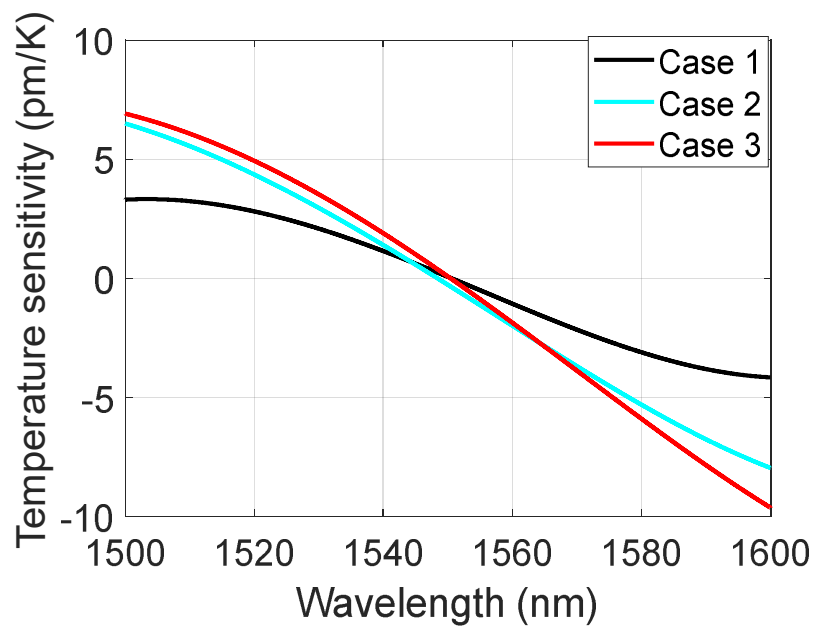

Fig. 4. Temperature insensitive MZI device's spectral response. Where for Case 1: $W_{2}=400 \mathrm{~nm}, W_{3}=400 \mathrm{~nm}, D C=40 \%, L=75.1 \mathrm{~mm}, \Delta L=64.4 \mathrm{~mm}$; Case 2. $W_{2}=500 \mathrm{~nm}, W_{3}=500 \mathrm{~nm}, D C=50 \%, L=62 \mathrm{~mm}, \Delta L=42.5 \mathrm{~mm}$; Case 3. $W_{2}=600 \mathrm{~nm}, W_{3}=600 \mathrm{~nm}, D C=60 \%, L=50.6 \mathrm{~mm}, \Delta L=23.5$ $\mathrm{mm}$

\section{CONCLUSION}

In this work, we have presented a design of a temperature insensitive Mach-Zehnder interferometer, in by introducing a temperature-compensating segment via tailored subwavelength gratings. The proposed device is also insensitive to the variations of its arm length of the waveguide $(L)$ up to the value of $\pm 0.35 \mathrm{~mm}$. The overall temperature sensitivity of the device $\pm 4 \mathrm{pm} /{ }^{\circ} \mathrm{K}$ was simulated for a $100 \mathrm{~nm}$ bandwidth in the $1550 \mathrm{~nm}$ wavelength region.

\section{ACKNOWLEDGEMENTS}

This work is supported by the Commonwealth Scholarship Commission, United Kingdom; European Union Horizon 2020 research and innovation program under the Marie Skłodowska-Curie Grant (734331); Spanish Ministry of Science, Innovation and Universities (TEC2015-71127-C2-1$\mathrm{R}$ and IJCI-2016-30484), and Community of Madrid (SINFOTON2-CM S2018/NMT-4326). Umair Ahmed Korai acknowledge the dept. of Telecom. Engineering, Mehran University of Engineering and Technology, Jamshoro, 76062, Pakistan.

\section{REFERENCES}

[1] A. Herrero-Bermello, A. V. Velasco, H. Podmore, P. Cheben, J. H. Schmid, S. Janz, M. L. Calvo, D. X. Xu, A. Scott, and P. Corredera, "Temperature dependence mitigation in stationary Fourier-transform on-chip spectrometers," Optics Letters, vol. 42, no. 11, pp. 2239-2242, 2017.

[2] G. Cocorullo, I. Rendina, "Thermo-optical modulation at $1.5 \mu \mathrm{m}$ in silicon etalon," Electronics Letters, vol. 28, no. 1, pp. 83-85, 1992.

[3] M. S. Rasras, D. M. Gill, S. S. Patel, K.-Y. Tu, Y.-K. Chen, A. E. White, A. T. Pomerene, D. N. Carothers, M. J. Grove, D. K. Sparacin, J. Michel, M. A. Beals, and L. C. Kimerling, "Demonstration of a fourth-order pole-zero optical filter integrated using CMOS processes," IEEE/OSA Journal of Lightwave Technology, vol. 25, no. 1, pp. 87-92, 2007.

[4] J. H. Schmid, M. Ibrahim, P. Cheben, J. Lapointe, S. Janz, P. J. Bock, A. Densmore, B. Lamontagne, R. Ma, W. N. Ye, and D. X. Xu, "Temperature-independent silicon subwavelength grating waveguides," Optics Letters, vol. 36, no. 11, pp. 2110-2112, 2011.

[5] T. Lipka, L. Moldenhauer, J. Müller, H. K. Trieu, "Athermal and wavelength-trimmable photonic filters based on TiO2-cladded amorphous-SOI", Optics Express, vol. 23, no. 15, pp. 20075-20088, 2015.

[6] B. Guha, A. Gondarenko, and M. Lipson, "Minimizing temperature sensitivity of silicon Mach-Zehnder interferometers," Optics Express, vol. 18, no. 3, pp. 1879-1887, 2010.

[7] M. Uenuma, T. Moooka, "Temperature-independent silicon waveguide optical filter," Optics Letters, vol. 34, no. 5, pp. 599-601, 2009.

[8] P. Cheben, R. Halir, J. H. Schmid, H. A. Atwater, D. R. Smith, "Subwavelength integrated photonics", Nature, vol. 560, no. 7720, pp. 565-572, Aug. 2018. 
